\title{
Clinical Study \\ Differentiated Thyroid Cancer with Extrathyroidal Extension: Prognosis and the Role of External Beam Radiotherapy
}

\author{
Michael A. Sia, ${ }^{1,2}$ Richard W. Tsang, ${ }^{3,4}$ Tony Panzarella, ,, 6 and James D. Brierley ${ }^{3,4}$ \\ ${ }^{1}$ Department of Radiation Oncology, Tom Baker Cancer Centre, Calgary, AB, Canada T2N 4N2 \\ ${ }^{2}$ Department of Radiation Oncology, University of Calgary, Calgary, AB, Canada T2N 4N1 \\ ${ }^{3}$ Department of Radiation Oncology, Princess Margaret Hospital, Toronto, ON, Canada M5G 2M9 \\ ${ }^{4}$ Department of Radiation Oncology, University of Toronto, Toronto, ON, Canada M5S 3E2 \\ ${ }^{5}$ Department of Biostatistics, Princess Margaret Hospital, University of Toronto, Toronto, ON, Canada M5S 2M9 \\ ${ }^{6}$ Dalla Lana School of Public Health, University of Toronto, Toronto, ON, Canada M5T 3M7
}

Correspondence should be addressed to James D. Brierley, james.brierley@rmp.uhn.on.ca

Received 3 July 2009; Accepted 2 March 2010

Academic Editor: Jeremy L. Freeman

Copyright ( 2010 Michael A. Sia et al. This is an open access article distributed under the Creative Commons Attribution License, which permits unrestricted use, distribution, and reproduction in any medium, provided the original work is properly cited.

\begin{abstract}
A study was performed to identify variables that affected cause-specific survival (CSS) and local relapse-free rate (LRFR) in patients with differentiated thyroid cancer (DTC) and extrathyroid extension (ETE) and to examine the role of external beam radiotherapy (XRT). Prognostic factors were similar to those found in studies of all patients with DTC. In patients with postoperative gross residual disease treated with radiotherapy, 10 -year CSS and LRFR were $48 \%$ and $90 \%$. For patients with no residual or microscopic disease, 10 -year CSS and LRFR were $92 \%$ and $93 \%$. In patients older than 60 years with T3 ETE but no gross residual disease postoperatively there was an improved LRFR at 5 years of $96 \%$, compared to $87.5 \%$ without XRT $(P=.02)$. Patients with gross ETE benefit from XRT and there may be a potential benefit in reducing locoregional failure in patients over 60 years with minimal extrathyroidal extension (T3).
\end{abstract}

\section{Introduction}

The role of radiotherapy in the management of differentiated thyroid cancer remains controversial with the majority of patients treated with thyroidectomy and radioactive iodine alone [1]. However, there is a subset of patients who are at high risk for local recurrence who may benefit from adjuvant external radiation therapy. The difficulty in determining the optimal management of patients is the lack of randomized control trials and the reliance on single institution prospective databases and retrospective reviews, complicated by changes in clinical practice, pathology assessment, and surgical techniques over time. Despite this, the British Thyroid Association recommends XRT in patients over 60 years of age with extensive extranodal spread after optimal surgery, even in the absence of evident residual disease [2], and the American Thyroid Association guidelines have recommended that "The use of external beam irradiation should be considered in patients over age of 45 with grossly visible extrathyroidal extension (T4a and T4b) at the time of surgery and a high likelihood of microscopic residual disease" [3].

Several factors have been identified to be prognostic in determining survival. These factors include age, tumor size, grade, presence of macroscopic disease after surgical resection, and presence of distant metastases $[4,5]$. Additionally extrathyroidal extension (ETE) has been identified as carrying a higher risk for local recurrence $[4,6,7]$. The definition of the AJCC and UICC T Category for thyroid cancer changed in 2002 and reflects the importance of varying degrees of ETE $[8,9]$. There are three different levels of extent. T3 represents minimal ETE to sternothyroid or perithyroid tissue or any tumor greater than $4 \mathrm{~cm}$. T4a includes invasion into subcutaneous soft tissue, larynx, trachea, esophagus, and recurrent laryngeal nerve. $\mathrm{T} 4 \mathrm{~b}$ includes invasion into prevertebral fascia, mediastinal 
vessels, or encasement of the carotid artery. In a retrospective review of 1067 patients with ETE treated by surgery alone, Ito et al. reported that minimal ETE (T3) did not have a deleterious effect on relapse-free survival (RFS) compared to no extrathyroidal extension, but that massive ETE (T4) did [10].

An analysis of a retrospective study from Princess Margaret Hospital $(\mathrm{PMH})$ has previously shown that patients over the age of 60 with ETE and no gross residual disease after surgery had significantly improved 10-year cause-specific survival (CSS) $(81.0 \%$ versus $64.6 \%, P=.04)$ and 10 year local relapse-free rates (LRFRs) (86.4\% versus $65.7 \%$, $P=.04)$ following adjuvant external radiation therapy [11]. Patients under 60 had improved local control, but not survival. The indications for radiotherapy at $\mathrm{PMH}$ have changed and have been refined over the years. Currently, the indications for XRT are patients over the age of 45 years with T4a or T4b tumors, or gross residual disease following thyroidectomy. Previously XRT was considered in all patients with ETE, but radiotherapy is now not advised for patients with T3 tumors (minimal ETE). This study is a retrospective review performed to further assess the role of XRT in patients with ETE and identify prognostic factors for local relapse-free rates and cause-specific survival in patients with differentiated thyroid cancer and ETE that might help more clearly to define who are at high risk and may benefit from XRT and to test the hypotheses that young patients with T4b disease benefited from XRT while older patients with T3 disease did not.

\section{Materials and Methods}

Following local ethics review board approval, a retrospective chart review of 323 patients with differentiated thyroid carcinoma (papillary or follicular) and known ETE, referred for adjuvant management and registered between January 1958 and December 1999, was conducted. The identification of 369 potentially eligible patients was previously described by Brierley et al. [11]. The pathology slides of all patients were reviewed at $\mathrm{PMH}$ on referral. As in our previous study, because of changes in the distribution of patients with follicular as opposed to papillary thyroid cancer, presumably due to changes in pathologists interpretation, patients with papillary or follicular tumors were analyzed together [11]. Of the 369 patients, nine were not considered due to difficulty in obtaining the source documentation for this chart review, and a total of 37 patients were excluded because they were referred for recurrent disease, for followup with primary treatment elsewhere, and for second opinion, or they had distant metastatic disease at presentation.

The sample size was not based on prestudy considerations of statistical power but on the available number of patients within the relevant time period. Cause-specific survival (CSS) and local relapse-free rates (LRFRs) were calculated by the cumulative incidence method. LRFR was defined as freedom from relapse in the neck, either thyroid bed or cervical lymph nodes, based on clinical examination or diagnostic imaging. Using the cumulative incidence method, competing risks were not censored, but acknowledged in the calculation. For CSS, the competing event was death from among other causes. For LRFR, competing events included deaths or metastatic failures

Univariate log-rank testing and multivariate Cox proportional hazards regression modeling analysis were performed on prognostic variables; this included the effect of treatment. Variables examined were histology, gender, age, tumor size, $\mathrm{T}$ category, differentiation, nodal involvement, postoperative status, multifocality, margin status, and lymphovascular involvement. In accordance with previous analyses from our institution, age was analyzed as a continuous variable and on a categorical basis $(<45,45-60,>60)[5,11,12]$. Tumor size was recorded and analyzed as a categorical variable, not a discrete variable; as in previous analyses, the categorical variables were $<1 \mathrm{~cm}, \geq 1 \mathrm{~cm}$ and $<4 \mathrm{~cm}$, and $\geq 4 \mathrm{~cm}$ as per earlier editions of TNM. Extent of ETE was categorized according to TNM 6th edition [8,9]. Patients with metastatic disease on first posttherapy radioactive iodine scan were considered to have metastases at time of presentation. Followup data was obtained through a review of patient charts and electronic records. For patients that were not routinely followed at $\mathrm{PMH}$, questionnaires were sent to attending physicians, followup phone calls were made to attending physicians, and cancer registry data was reviewed.

As a result of multiple significance testing (from the prognostic factor analysis and several subgroup analyses, on two endpoints) the Type I error is inflated so it is plausible that some of the effects presented as statistically significant are spurious. However, as it has been argued that the adjustment of $P$-values with the Bonferroni method creates more problems than it solves [13], the $P$-values in this report are unadjusted for multiple comparisons. Moreover, given the limited sample size, our subgroup analyses have low power (i.e., an inflated Type II error) to detect statistically significant differences. Competing risk failure probabilities and plots were produced with the cmprsk package using version 2.6.0 in R. All other analyses were performed using SAS version 9.1 .

\section{Results}

3.1. Patient Characteristics. The distribution of patient characteristics, candidate prognostic factors and treatments, are summarized in Table 1. The median followup among living cases $(n=235)$ was 10.8 years with a range from 0.6 to 39.5 years. The overall 5- and 10-year local relapsefree rates are 92.5\% (SE 0.02\%) and 91.7\% (SE 0.02\%), respectively. Of 323 patients, 56 have failed locally or regionally. The overall 5 - and 10-year cause-specific survival rates are $89.8 \%$ (SE $0.03 \%$ ) and $85.4 \%$ (SE $0.04 \%$ ), respectively (Figure 1). Of 323 patients, 88 died; 46 died of disease and 37 died of other causes. In 5 cases, the cause of death was unclear. Of patients who had data regarding site of disease at time of death, 71\% had distant metastasis, while $29 \%$ had local regional disease alone. 
TABle 1: Distribution of candidate prognostic factors and treatments.

\begin{tabular}{|c|c|c|}
\hline & \multicolumn{2}{|c|}{ All $N=323$} \\
\hline & $N$ & $\%$ \\
\hline \multicolumn{3}{|l|}{ Histology } \\
\hline Follicular & 60 & 18.58 \\
\hline Papillary & 261 & 80.80 \\
\hline \multicolumn{3}{|l|}{ Gender } \\
\hline Female & 239 & 73.99 \\
\hline Male & 84 & 26.01 \\
\hline \multicolumn{3}{|l|}{ Age } \\
\hline$<45$ & 134 & 41.49 \\
\hline $45-60$ & 95 & 29.41 \\
\hline$>60$ & 93 & 28.79 \\
\hline \multicolumn{3}{|l|}{ Size } \\
\hline$<1 \mathrm{~cm}$ & 20 & 6.19 \\
\hline $1-4 \mathrm{~cm}$ & 187 & 57.89 \\
\hline$>4 \mathrm{~cm}$ & 72 & 22.29 \\
\hline \multicolumn{3}{|l|}{ Differentiation } \\
\hline Moderate & 37 & 11.46 \\
\hline Poor & 52 & 16.10 \\
\hline Well & 98 & 30.34 \\
\hline \multicolumn{3}{|l|}{ Nodal involvement } \\
\hline No & 148 & 45.82 \\
\hline Yes & 149 & 46.13 \\
\hline \multicolumn{3}{|l|}{ Postoperative status } \\
\hline Gross & 28 & 8.67 \\
\hline No residual/microscopic & 259 & 80.19 \\
\hline \multicolumn{3}{|l|}{ Multifocal } \\
\hline No & 187 & 57.89 \\
\hline Yes & 119 & 36.84 \\
\hline \multicolumn{3}{|l|}{ Margin status } \\
\hline Negative & 195 & 60.37 \\
\hline Positive & 92 & 28.48 \\
\hline \multicolumn{3}{|l|}{ LVI (Lymph vascular Invasion) } \\
\hline No & 87 & 26.93 \\
\hline Yes & 130 & 40.25 \\
\hline \multicolumn{3}{|l|}{ T category } \\
\hline 3 & 169 & 52.32 \\
\hline $4 \mathrm{a}$ & 91 & 28.17 \\
\hline $4 \mathrm{~b}$ & 58 & 17.96 \\
\hline \multicolumn{3}{|l|}{ Surgery } \\
\hline Biopsy & 22 & 6.81 \\
\hline Lobectomy & 64 & 19.81 \\
\hline Near total & 80 & 24.77 \\
\hline Radical & 7 & 2.17 \\
\hline Total & 146 & 45.20 \\
\hline \multicolumn{3}{|l|}{ RAI (Radioactive Iodine) } \\
\hline No & 65 & 20.12 \\
\hline Yes & 258 & 79.88 \\
\hline \multicolumn{3}{|l|}{ XRT } \\
\hline No & 115 & 35.60 \\
\hline Yes & 208 & 64.40 \\
\hline
\end{tabular}

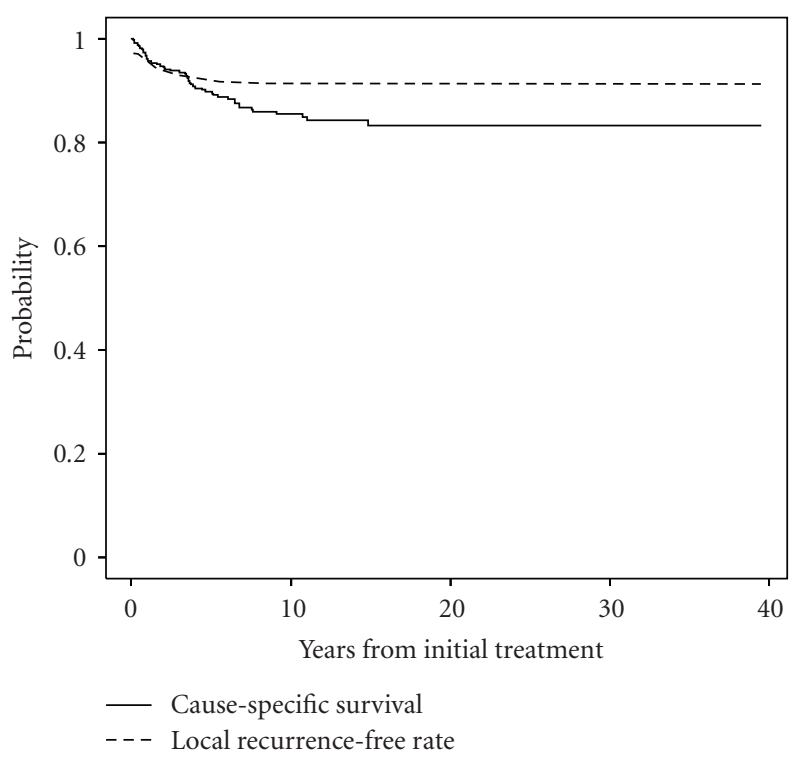

Figure 1: Cause-specific survival and local recurrence-free rate for patients with differentiated thyroid cancer and extrathyroidal extension. The overall 5- and 10-year cause-specific survivals are $89.8 \%$ (SE $0.03 \%$ ) and $85.4 \%$ (SE $0.04 \%$ ), respectively. The overall 5 - and 10 -year local relapse-free rates are $92.5 \%$ (SE 0.02\%) and $91.7 \%$ (SE 0.02\%), respectively.

3.2. Prognostic Factor and Effects of Treatment Analyses: Baseline Variables. The candidate prognostic factors and effects of treatment on cause-specific survival and local relapse-free rates were subjected to univariate and multivariate analyses. Local invasion of subsites (i.e., larynx, trachea, esophagus, prevertebral fascia, mediastinal vessels, etc.) for T4a and T4b was collected but not included in the analysis as the number of events for each subsite was small.

Factors found to be significant for a worse cause-specific survival on univariate analysis are listed in Table 2. Unexpectedly patients with multifocal disease had better causespecific survival. With respect to treatment, no difference in cause-specific survival was observed in patients receiving radioactive iodine ablation. Patients who underwent near total thyroidectomy had better outcomes. The factors which carried a statistically significant association with outcome on univariate analysis for local relapse rates are listed in Table 2. $\mathrm{T}$ category was not a statistically significant prognostic factor for local relapse-free rates. Treatment modalities (radioactive iodine ablation, XRT, and surgery) did not influence overall local relapse-free rates. However, surgery was of borderline significance.

Multivariate analysis was performed on the majority of candidate prognostic factors found to be significant on univariate testing (Table 3 ). The factors statistically significant for improved cause-specific survival were postoperative status (no residual), papillary histology, and younger age$<45$. The presence of multifocal disease remained a significant favorable prognostic factor for cause-specific survival. Smaller tumor size $(<1 \mathrm{~cm}$ and $1-4 \mathrm{~cm})$ and postoperative status (no residual) were significant for improved local 
TABLE 2: Univariate analysis and Log-rank $P$ value: summary.

\begin{tabular}{lcc}
\hline Variable & CSS & LRFR \\
\hline Histology $(N=321)$ & $<0.0001$ & 0.15 \\
Gender $(N=323)$ & 0.51 & $<.39$ \\
Age $(N=322)$ & $<0.0001$ & $<0.0001$ \\
Differentiation $(N=187)$ & 0.002 & 0.034 \\
Tumor size $(N=279)$ & $<0.0001$ & $<0.0004$ \\
Postoperative status $(N=309)$ & 0.74 & 0.0001 \\
Neck metastasis $(N=297)$ & 0.009 & 0.96 \\
Multifocal $(N=306)$ & 0.005 & 0.65 \\
Margin status $(N=287)$ & 0.0006 & 0.09 \\
LVI $(N=217)$ & 0.014 & 0.24 \\
T category $($ worst of pathology/OR) $(N=322)$ & 0.62 & 0.13 \\
RAI $(N=323)$ & 0.01 & 0.71 \\
RT $(N=323)$ & 0.01 & 0.074 \\
SURGERY $(N=290)$ & & \\
\hline
\end{tabular}

relapse-free rates. There was a trend towards significance with age $(P=.09)$. There was no effect from T (T3, T4a, or T4b) category for CSS or LRFR on the multivariate model. No association was seen between treatment modalities and improved cause-specific survival or local relapse-free rates.

3.3. Subgroup Analyses. In 40 patients with postoperative gross residual disease treated with radiotherapy, 10-year CSS and LRFR were $48 \%$ and $90 \%$, respectively (Figure 2 ). Further subgroup analyses were performed in patients without gross residual disease after surgery, that is, no residual disease or microscopic residual. Patients were subdivided based on age (less than 45 years, 45-60 years, and greater than 60 years) and extent of extrathyroidal extension (T3, T4a, and T4b). Patients were also analyzed based on a combination of age and $\mathrm{T}$ category. XRT did not result in any significant difference in CSS for any subgroup. There was a trend in favor of improved LRFR in T3 tumors with XRT $(94.8 \%$ versus $87.3 \%$ at 5 years, $P=.08$ ). There was a significant benefit in patients over 45 with $\mathrm{T} 3$ disease treated with XRT at 5 years. The LRFRs were 96.8\% versus 90\%, $P=$ .03 , (Figure 3 ), although in this subgroup there was a trend towards more use of RAI in patients who also had XRT. There was however no significant difference in LRFR for patients between 45 and 60, with the benefit being in those over 60 (96\% versus $87.5 \%, P=.02$, Figure 4$)$, and in this subgroup there was no difference in the use of RAI although the number of patients was small. There was no benefit with XRT in T3 patients less than 45. Also there was no apparent benefit to XRT for local relapse-free rates in T4 tumors in those less than 45 years or greater than 60 years, but the numbers were relatively small.

\section{Discussion}

This study demonstrates a potential benefit to radiotherapy in reducing locoregional failure in patients over 60 years

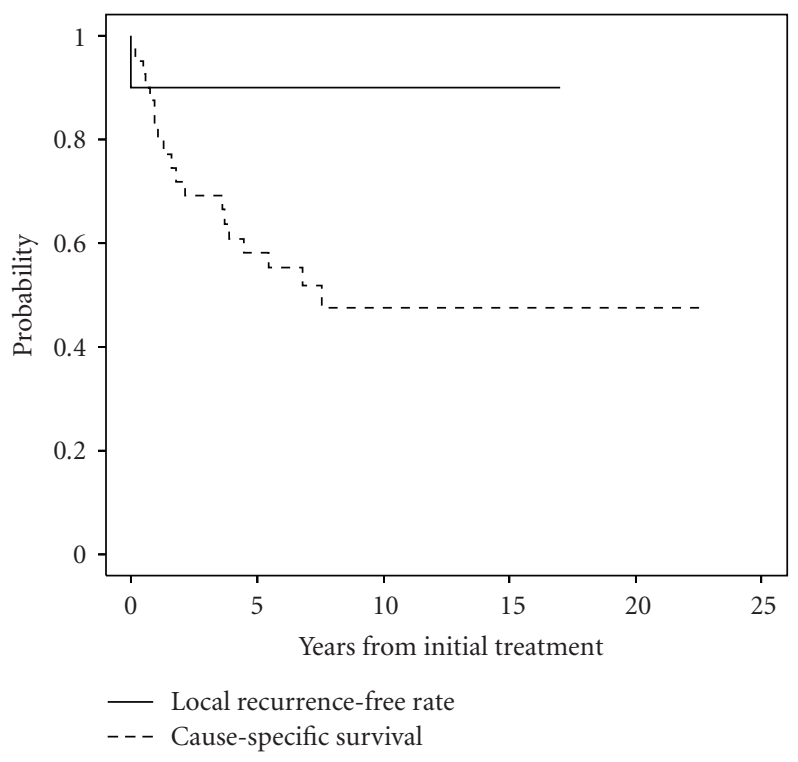

FIGURE 2: Cause-specific survival and local recurrence-free rate for patients with gross residual disease treated with RT; 10-year CSS and LRFR were $48 \%$ and $90 \%$, respectively.

with minimal extrathyroidal extension (T3). The role of XRT in differentiated thyroid cancer remains unclear due to the lack of prospective randomized studies and the reliance on evidence from retrospective studies. The use of XRT in the management of thyroid cancer varies among centers, but several retrospective studies have shown a benefit with radiotherapy [14-22] and it is now recommended by several treatment guidelines in certain high-risk groups [2, $3,23,24]$. Our current indications for adjuvant radiotherapy include patients over the age of 45 years with gross residual disease or over 45 years with clinical gross extrathyroidal invasion (T4), but not for patients with T3 tumors (minimal ETE). 
TABLE 3: Multivariate analysis summary—Cox proportional hazards modeling. $(* *)$

\begin{tabular}{|c|c|c|c|c|}
\hline \multirow[b]{2}{*}{ Variable } & \multicolumn{2}{|c|}{ CSS ( $N=253$ with 26 events $)$} & \multicolumn{2}{|c|}{ LRFR ( $N=263$ with 41 events $)$} \\
\hline & $\begin{array}{l}\text { Hazard ratio (HR) }(95 \% \\
\text { confidence interval (CI)) }\end{array}$ & $P$ value & HR (95\% CI) & $P$ value \\
\hline Histology & $8.7(3.6-21.0)$ & $<.0001$ & & \\
\hline Age & $3.1(1.2-8.2)$ & .02 & $1.8(0.9-3.7)$ & .09 \\
\hline \multicolumn{5}{|l|}{ Differentiation $(*)$} \\
\hline Tumor size & $1.8(0.7-4.6)$ & .25 & $2.1(1.04-4.1)$ & .04 \\
\hline Postoperative status & $7.1(2.6-19.6)$ & .0001 & $4.6(2.2-9.8)$ & $<.0001$ \\
\hline Neck metastasis & & & $1.4(0.8-2.7)$ & .26 \\
\hline Multifocal & $0.3(0.1-0.9)$ & .026 & & \\
\hline Margin status & $1.3(0.5-3.4)$ & .56 & & \\
\hline \multicolumn{5}{|l|}{$\operatorname{LVI}(*)$} \\
\hline T category (worst of pathology/OR) & $1.2(0.5-3.3)$ & .68 & & \\
\hline RAI & $0.5(0.1-1.9)$ & .29 & $0.9(0.3-2.4)$ & .76 \\
\hline RT & $0.9(0.3-2.8)$ & .81 & $1.4(0.7-2.9)$ & .36 \\
\hline SURGERY & $0.6(0.2-1.7)$ & .36 & $1.03(0.5-2.0)$ & .93 \\
\hline
\end{tabular}

$(*)$ Not considered in the multivariate analysis because of the extent of missing data.

Note. Cox model was stratified by 3 approximately equal time periods: 1958-1971, 1972-1985, and 1986-1998 [11].

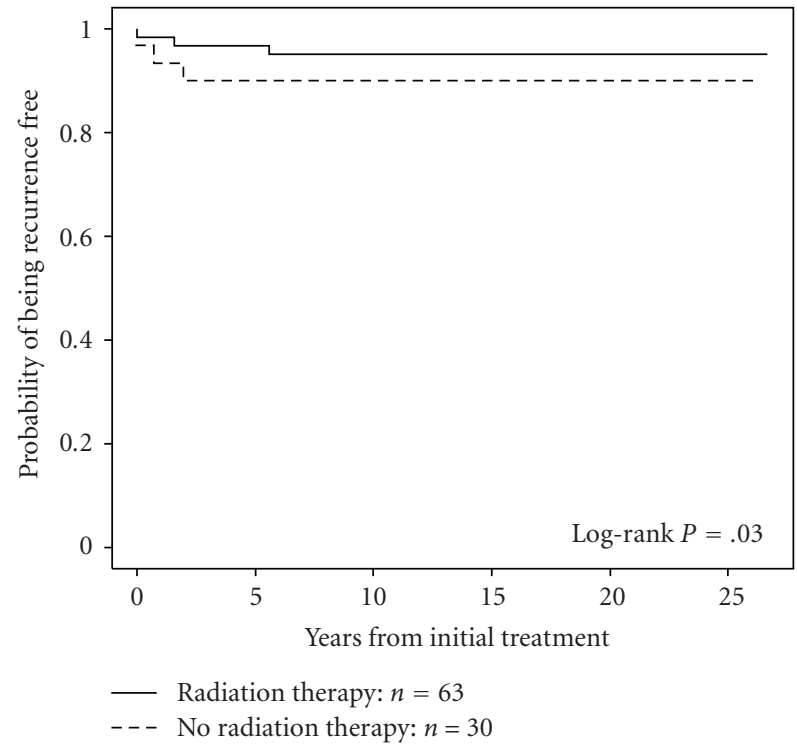

FIGURE 3: Local recurrence-free rate for patients over 45 years of age with T3 tumors treated with and without RT, $96.8 \%$ versus $90 \%$ at 5 years, respectively, $P=.03$.

Age has been shown to be a significant factor for determining prognosis in differentiated thyroid cancer that is the only tumor type in which the AJCC and UICC use age for stage determination. Our previous study in [11] and another in [25] showed a greater worsening of outcome for patients over 60 years of age. Extrathyroidal extension has also been identified as a prognostic factor carrying a higher risk for local recurrence [5-7]. Following our recent retrospective analysis of adjuvant radiotherapy although no benefit to

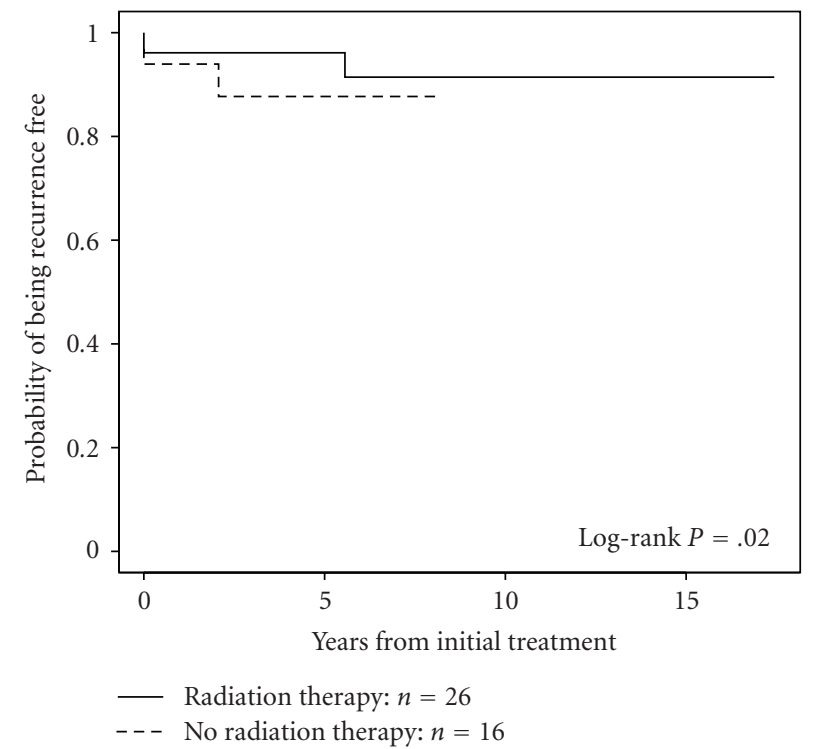

FIGURE 4: Local recurrence free rate for patients over 60 years of age with T3 tumors treated with and without RT, $96 \%$ versus $87.5 \%$, at 5 years, respectively, $P=.02$.

adjuvant radiotherapy was seen in patients less than 60 years with ETE, we speculated that, since the definition of ETE encompasses a large range of disease extent from minimal invasion of perithyroidal fat to invasion of the prevertebral fascia, there might still be a benefit to adjuvant radiotherapy in carefully selected high-risk patients under 60 years. Conversely, we also speculated that there might be a subset of patients over 60 years with minimal ETE that did not benefit from adjuvant radiotherapy. 
Journal of Thyroid Research

We were unable to demonstrate a benefit with radiotherapy for cause-specific survival and local relapse-free rates in T4 tumors in those less than 45 years, but the number of events was small. Also no benefit was seen with radiotherapy in those over 60 years with T4 disease and also the number of events was small. There was a significant benefit for local relapse-free rates, but not for cause-specific survival in patients over 60 years with T3 disease treated with radiotherapy. This benefit in patients with T3 disease was unexpected especially in light of the findings of Ito et al. that minimal ETE did not carry a poor prognosis for RFS for patients over the age of 45 [10], although they did not report whether there was a difference in RFS in T3 tumors by age greater or less than 60 . It could be speculated that for patients greater than 60 there is a difference in outcome for patients with limited ETE (seen only microscopically), compared with those tumors with invasion into sternothyroid or perithyroid tissues noted at the time of surgery. The potential benefit of XRT if it really exists is small in T3 disease and the data should not be overinterpreted given that this is a retrospective review of patients seen over a long-time period with changing practices in surgery, radiotherapy, and the use of iodine. Another caveat is that multiple statistical comparisons were explored in subgroups, which increased the chances of finding a significant difference by chance alone.

The benefit of radiotherapy in differentiated thyroid cancers with gross residual disease has been shown in several studies $[14,15,17,18]$. Chow and colleagues [26] demonstrated that extent of postsurgical residual disease was associated with cause-specific survival and that, when this factor was included in multivariate analysis, it overrode the other prognostic factors (size of tumor, extrathyroidal extension, lymph node metastases, and type of surgery). Analysis from our institution has also found postoperative presence of macroscopic residual disease to be a significant factor for 10-year local relapse-free rates and cause-specific survival [5]. In this study, patients with postoperative gross residual disease treated with radiotherapy had 10 -year causespecific survival and local relapse-free rates of $48 \%$ and $90 \%$, respectively, demonstrating that even if disease is controlled in the neck there is a high risk of death from distant metastatic disease.

\section{Conclusions}

Prognostic factors for patients with ETE are similar to those of any patient with differentiated thyroid cancer. Although radiotherapy is of benefit in patients with gross residual disease, we were unable to define a subset of patients less than 45 years who benefited from adjuvant radiotherapy. There may be a potential benefit to radiotherapy in reducing locoregional failure in patients over 60 years with minimal extrathyroidal extension (T3). There remains uncertainty in how to select high-risk patients for radiotherapy. The potential benefit needs to be balanced against the generally excellent outcomes with surgery and radioactive iodine as well as the expected side effects of radiotherapy, although these may be reduced with modern radiation techniques [27].

\section{Disclosure}

The authors have no competing interests.

\section{References}

[1] N. Lee and M. Tuttle, "The role of external beam radiotherapy in the treatment of papillary thyroid cancer," EndocrineRelated Cancer, vol. 13, no. 4, pp. 971-977, 2006.

[2] British Thyroid Association, Guidelines for the Management of Thyroid Cancer in Adults, Royal College of Physicians, London, UK, 2006.

[3] D. S. Cooper, G. M. Doherty, B. R. Haugen, et al., "Management guidelines for patients with thyroid nodules and differentiated thyroid cancer," Thyroid, vol. 16, no. 2, pp. 109141, 2006.

[4] J. D. Brierley and S. L. Asa, "Thyroid cancer," in Prognostic Factors in Cancer, M. K. Gospodarowicz, B. O'Sullivan, and L. H. Sobin, Eds., John Wiley \& Sons, Hoboken, NJ, USA, 2006.

[5] R. W. Tsang, J. D. Brierley, W. J. Simpson, T. Panzarella, M. K. Gospodarowicz, and S. B. Sutcliffe, "The effects of surgery, radioiodine, and external radiation therapy on the clinical outcome of patients with differentiated thyroid carcinoma," Cancer, vol. 82, no. 2, pp. 375-388, 1998.

[6] B. Cady, R. Rossi, I. Hay, K. H. Cohn, and N. W. Thompson, "An expanded view of risk-group definition in differentiated thyroid carcinoma," Surgery, vol. 104, no. 6, pp. 947-953, 1988.

[7] I. D. Hay, "Papillary thyroid carcinoma," Endocrinology and Metabolism Clinics of North America, vol. 19, no. 3, pp. 545576, 1990.

[8] L. H. Sobin and C. Wittekind, Eds., TNM Classification of Malignant Tumours, John Wiley \& Sons, New York, NY, USA, 6th edition, 2002.

[9] F. L. Greene, et al., AJCC Cancer Staging Manual, Springer, New York, NY, USA, 6th edition, 2002.

[10] Y. Ito, C. Tomoda, T. Uruno, et al., "Minimal extrathyroid extension does not affect the relapse-free survival of patients with papillary thyroid carcinoma measuring $4 \mathrm{~cm}$ or less over the age of 45 years," Surgery Today, vol. 36, no. 1, pp. 12-18, 2006.

[11] J. Brierley, R. Tsang, T. Panzarella, and N. Bana, "Prognostic factors and the effect of treatment with radioactive iodine and external beam radiation on patients with differentiated thyroid cancer seen at a single institution over 40 years," Clinical Endocrinology, vol. 63, no. 4, pp. 418-427, 2005.

[12] W. J. Simpson, S. E. McKinney, J. S. Carruthers, M. K. Gospodarowicz, S. B. Sutcliffe, and T. Panzarella, "Papillary and follicular thyroid cancer. Prognostic factors in 1,578 patients," American Journal of Medicine, vol. 83, no. 3, pp. 479488, 1987.

[13] T. V. Perneger, "What's wrong with Bonferroni adjustments," British Medical Journal, vol. 316, no. 7139, pp. 1236-1238, 1998.

[14] M. Tubiana, E. Haddad, and M. Schlumberger, "External radiotherapy in thyroid cancers," Cancer, vol. 55, no. 9, supplement, pp. 2062-2071, 1985.

[15] L.-T. Wu, S. D. Averbuch, D. W. Ball, A. De Bustros, S. B. Baylin, and W. P. McGuire III, "Treatment of advanced medullary thyroid carcinoma with a combination of cyclophosphamide, vincristine, and dacarbazine," Cancer, vol. 73, no. 2, pp. 432-436, 1994. 
[16] P. Phlips, C. Hanzen, G. Andry, P. Van Houtte, and J. Fruuling, "Postoperative irradiation for thyroid cancer," European Journal of Surgical Oncology, vol. 19, no. 5, pp. 399-404, 1993.

[17] O. Esik, G. Nemeth, and J. Eller, "Prophylactic external irradiation in differentiated thyroid cancer: a retrospective study over a 30-year observation period," Oncology, vol. 51, no. 4, pp. 372-379, 1994.

[18] M. E. A. O'Connell, R. P. A'Hern, and C. L. Harmer, "Results of external beam radiotherapy in differentiated thyroid carcinoma: a retrospective study from the Royal Marsden Hospital," European Journal of Cancer A, vol. 30, no. 6, pp. 733-739, 1994.

[19] J. Farahati, C. Reiners, M. Stuschke, et al., "Differentiated thyroid cancer: impact of adjuvant external radiotherapy in patients with perithyroidal tumor infiltration (stage pT4)," Cancer, vol. 77, no. 1, pp. 172-180, 1996.

[20] T.-H. Kim, D.-S. Yang, K.-Y. Jung, C.-Y. Kim, and M.-S. Choi, "Value of external irradiation for locally advanced papillary thyroid cancer," International Journal of Radiation Oncology Biology Physics, vol. 55, no. 4, pp. 1006-1012, 2003.

[21] K. C. Keum, Y. G. Suh, W. S. Koom, et al., "The role of postoperative external-beam radiotherapy in the management of patients with papillary thyroid cancer invading the trachea," International Journal of Radiation Oncology Biology Physics, vol. 65, no. 2, pp. 474-480, 2006.

[22] K. M. Meadows, R. J. Amdur, C. G. Morris, D. B. Villaret, E. L. Mazzaferri, and W. M. Mendenhall, "External beam radiotherapy for differentiated thyroid cancer," American Journal of Otolaryngology, vol. 27, no. 1, pp. 24-28, 2006.

[23] S. Sherman, et al., "Thyroid carcinoma," in Practice Guidelines in Onclogy, National Comprehensive Cancer Network, 2007, http://www.nccn.org/index.asp.

[24] F. Pacini, M. Schlumberger, H. Dralle, et al., "European consensus for the management of patients with differentiated thyroid carcinoma of the follicular epithelium," European Journal of Endocrinology, vol. 154, no. 6, pp. 787-803, 2006.

[25] E. L. Mazzaferri and S. M. Jhiang, "Long-term impact of initial surgical and medical therapy on papillary and follicular thyroid cancer," American Journal of Medicine, vol. 97, no. 5, pp. 418-428, 1994.

[26] S.-M. Chow, S. Yau, C.-K. Kwan, P. C. M. Poon, and S. C. K. Law, "Local and regional control in patients with papillary thyroid carcinoma: specific indications of external radiotherapy and radioactive iodine according to $\mathrm{T}$ and $\mathrm{N}$ categories in AJCC 6th edition," Endocrine-Related Cancer, vol. 13, no. 4, pp. 1159-1172, 2006.

[27] B. D. Rosenbluth, V. Serrano, L. Happersett, et al., "Intensitymodulated radiation therapy for the treatment of nonanaplastic thyroid cancer," International Journal of Radiation Oncology Biology Physics, vol. 63, no. 5, pp. 1419-1426, 2005. 


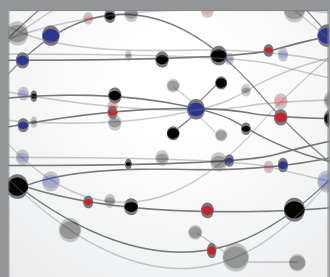

The Scientific World Journal
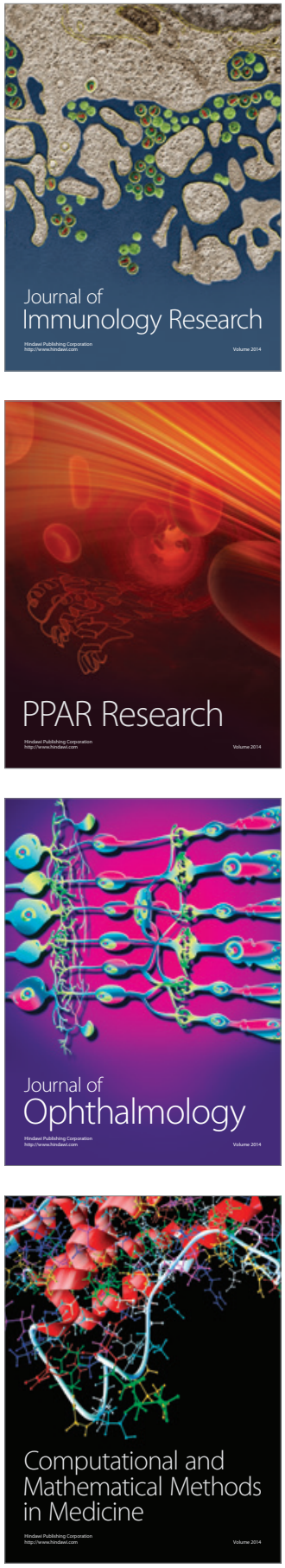

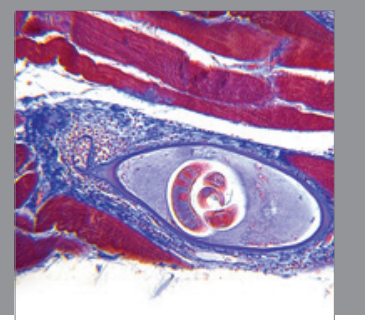

Gastroenterology

Research and Practice
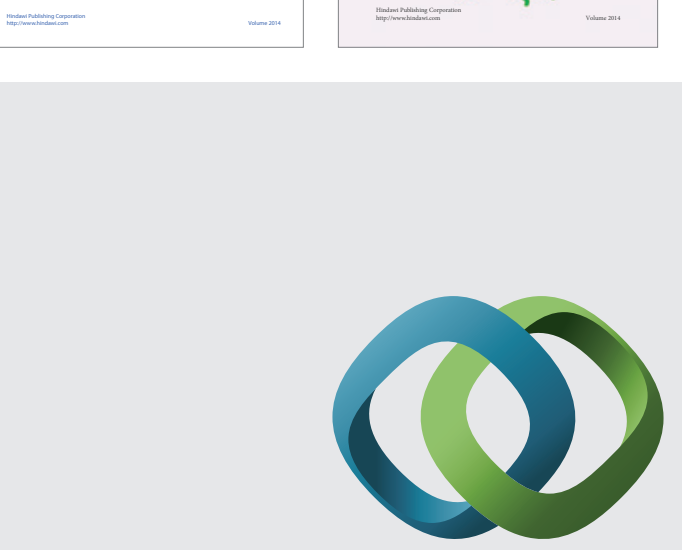

\section{Hindawi}

Submit your manuscripts at

http://www.hindawi.com
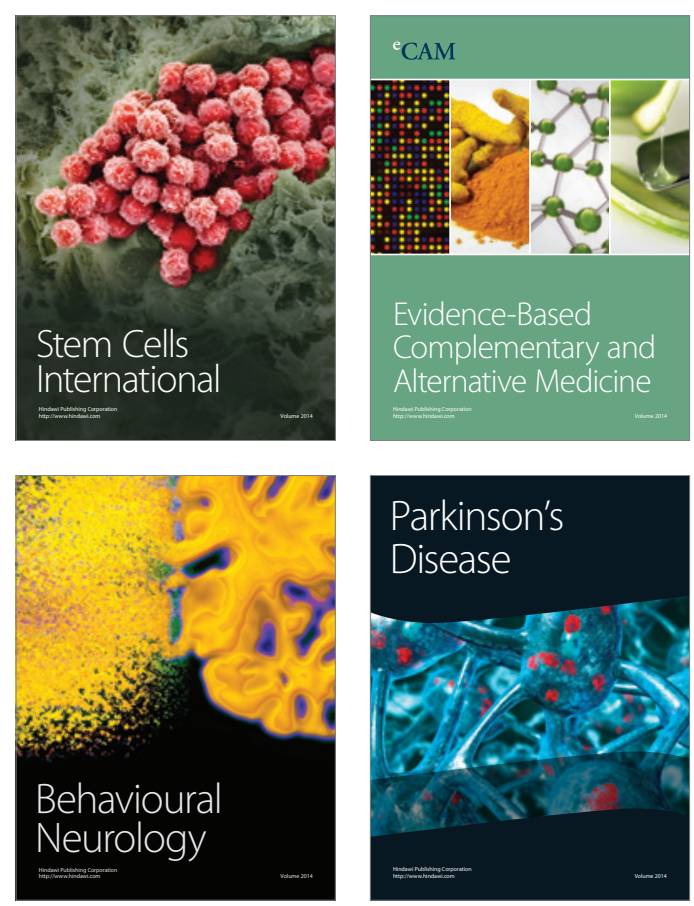

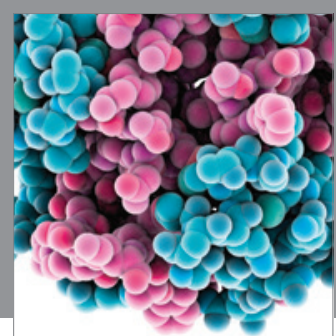

Journal of
Diabetes Research

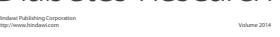

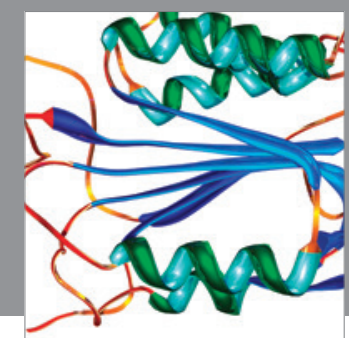

Disease Markers
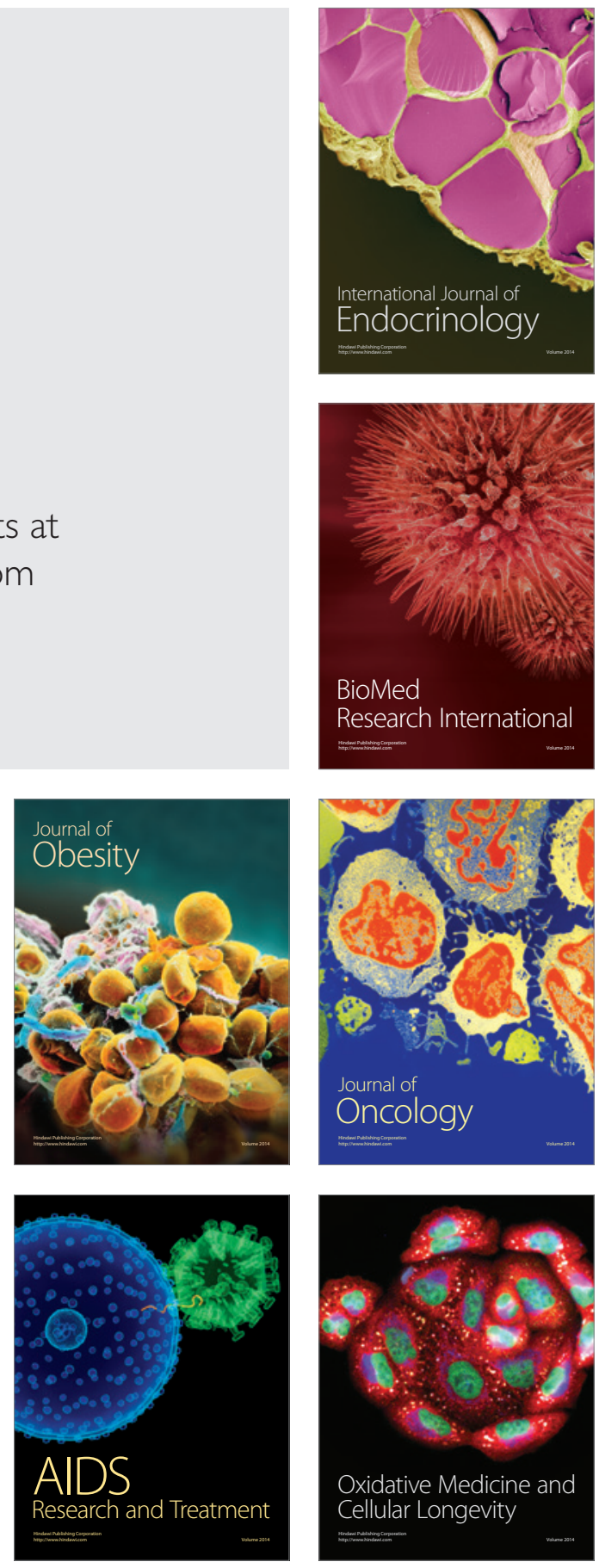